\title{
Egg-laying preference for ethanol involving learning has adaptive significance in Drosophila melanogaster
}

\author{
N. CADIEU, L. EL GHADRAOUI, and J.-C. CADIEU \\ Université Paul Sabatier, Toulouse, France
}

\begin{abstract}
To study the influence of negative or positive experience of the ingestion of ethanol in female Drosophila with the fast form of the Adh gene on the acceptance of a medium containing this substance as site for egg laying, flies raised on a medium without alcohol were kept (1) on a regular medium, (2) on a medium supplemented with ethanol, (3) on a medium supplemented with 4methylpyrazole (4-MP, an inhibitor of the enzyme $\mathrm{ADH}$ ), and (4) on a medium containing both ethanol and this inhibitor. Whereas the Drosophila exhibited no spontaneous egg-laying preference for ethanol, a preference was induced by the ingestion of this substance, which also led to an increase in the number of eggs laid. By contrast, ingestion of ethanol, associated with the inhibitor, reduced the number of eggs laid and led to an avoidance of ethanol-containing media. Flies treated with ethanol and 4-MP exhibited signs of sickness (reduction in locomotor activity and loss of balance). The egg-laying preference for the ethanol results from learning linked to the metabolic utilization of this alcohol. The adaptative significance of this learning is discussed.
\end{abstract}

It is now recognized that learning plays a role in the location and the acceptance of a host as egg-laying site in various species of insects. This has been demonstrated in various parasitoids as well as in frugivorous and herbivorous species (Henneman, Papaj, Figueredo, \& Vet, 1995; Hoffmann, 1985, 1988; Jaenike, 1982; Kaiser \& De Jong, 1995; Lewis \& Tumlinson, 1988; Papaj \& Prokopy, 1989; Prokopy, Averill, Cooley, \& Roitberg, 1982; Traynier, 1984; Vet, 1983). Learning has been invoked to explain the maintenance of genetic polymorphism, as well as the formation of host races giving rise to speciation (Jaenike, 1982; Maynard-Smith, 1966; Thorpe, 1963).

The role of habituation to repellent substances or other forms of nonassociative learning has been noted in regard to selection of egg-laying site. Associative learning was also demonstrated and, in this case, oviposition was frequently the reward involved in conditioning to odors, shapes, and colors (Kaiser \& De Jong, 1995; Papaj \& Prokopy, 1989; Prokopy et al., 1982; Traynier, 1984). Some studies have shown, however, that the recognition and acceptance of the egg-laying site may be influenced by other types of reinforcement (Papaj, 1986). Learning may also lead to long-lasting alterations in preferences (Hoffmann, 1988; Thorpe, 1963; Wardle \& Borden, 1985). The reversible nature of certain types of learning has been widely studied since it is regarded as a response to spatiotemporal fluctuations in the field (Iizuka \& Takasu, 1998; Papaj \& Prokopy, 1989; Vet, 1983).

We thank J. David for supplying the Drosophila strains. Correspondence should be addressed to N. Cadieu, Laboratoire d'Ethologie et de Psychologie Animale, Université Paul Sabatier, 118 route de Narbonne, 31062 Toulouse Cedex 4, France (e-mail: cadieu@cict.fr).
Drosophila melanogaster generally lay their eggs on fermented media (McKenzie \& Parsons, 1972), and in the wild, the main food source is represented by fermented fruit. Low concentrations of alcohol are tolerated and used as food (Cadieu, El Amrani, \& Cadieu, 1997; David, Boquet, Arens, \& Fouillet, 1976; DeltombeLietaert, Delcour, Lenelle-Monfort, \& Elens, 1979; El Ghadraoui, Cadieu, \& Cadieu, 1995; Heinstra, 1993). In Drosophila melanogaster, alcohol dehydrogenase (ADH) is the key enzyme for detoxification and metabolism of ethanol (David, 1977, 1978; David \& Bocquet, 1975). Its activity is controlled by the Adh gene, which exists in several allelic forms, the most common being the fast and slow alleles (high and low levels of activity).

Several studies have been conducted to find out whether there is a relationship between the genetic ability to metabolize ethanol and the response to the odor of alcohol by females or the choice of alcohol-containing medium as site for egg laying. The results have been somewhat contradictory (Cavener, 1979; El Amrani, Cadieu, \& Cadieu, 1991; Fuyama, 1976; Hoffmann \& Cohan, 1987; Hougouto, Lietaert, Libion-Mannaert, Feytmans, \& Elens, 1982; Van Delden \& Kamping, 1990). Despite the longstanding discussion of the role of learning in the adaptation of insects such as wasps, bees, butterflies, and flies to ecological conditions (Papaj \& Prokopy, 1989), few authors have taken into account the role of learning in the choice of alcohol-containing media by different genotypes of Drosophila.

However, over the last few decades, a large number of studies have been devoted to learning and its genetic control in this species (Davis, 1996; Dudai, 1977; Dura, Prat, \& Tully, 1993; Médioni, Cadieu, \& Vaysse, 1978; Tully, 
1996; Tully \& Quinn, 1985). Various forms of learning, such as habituation, sensitization, and conditioning, have been identified (for review, see Davis, 1996). Study of mutations involved in learning and memory have indicated a biochemical commonality of these various types of learning and the relationships between them in Drosophila melanogaster (Davis, 1996; Tully, 1996). However, in a recent publication, Dukas (1998) remarked on the relatively few studies on the adaptive value of associative learning in Drosophila, and he made mention of the ecological significance of this type of learning in the larvae of this species.

Some studies have shown that the preference for ethanol both as food and as a site for egg laying can be modified by learning. Habituation to repellent doses of ethanol in the egg-laying medium, as well as the influence of exposure to this alcohol in early life, has been demonstrated (Cadieu et al., 1997; El Amrani et al., 1991; Jaenike, 1982). It is possible, however, to reverse the food preference for alcohol-containing media of fast Drosophila learning. An aversion for ethanol is induced by treatment with an inhibitor of ADH, such as 4-methylpyrazole (4-MP). This aversion is accompanied by signs of sickness that suggest the existence of a punishment following the ingestion of ethanol (Cadieu, Cadieu, El Ghadraoui, Grimal, \& Lamboeuf, 1999). In adult Drosophila, pyrazole and its derivatives have been shown to mainly inhibit $\mathrm{ADH}$, which degrades ethanol into acetaldehyde, and partially inhibit ALDH, which degrades acetaldehyde into acetate (Heinstra \& Geer, 1991). In vivo, the action of 4-MP is seen mainly as an increase in the concentration of ethanol in tissues due to inhibition of $\mathrm{ADH}$; acetaldehyde is either not produced or is rapidly degraded to acetic acid by ALDH, which is only partially inhibited (Delmas, 1981).

The use of a specific inhibitor, 4-MP, of ADH effectively increases the level of ethanol to which the flies are exposed and has two advantages: (1) The modifications of preference will involve solely the ADH pathway (rather than other catabolic routes of ethanol; Heinstra, Geer, Seykens, \& Langevin, 1989), whose role in the adaptation of Drosophila to alteration in levels of ethanol in the host medium has been abundantly discussed; and (2) 4-MP leads to an elevation of ethanol levels in tissues, which will affect behavior via an internal action rather than by stimulating external chemoreceptors by high and repellent doses in the medium. The long-term effects of the contact of these receptors with a repellent substance are well known in insects (Blaney \& Simmonds, 1985).

The present study was designed to find out whether preference for an alcohol-containing medium as an egglaying site for Drosophila melanogaster stemmed from learning and whether this learning had an adaptive value with respect to the ability to metabolize ethanol via the $\mathrm{ADH}$ pathway. We investigated the effects of a positive or negative experience of ethanol on number and proportion of eggs laid on the alcohol medium in naive flies that were genetically able to detoxify this substance. The positive experience was obtained from previous ingestion of ethanol, while the negative one was obtained by prior ingestion of ethanol associated with the alcohol metabolism inhibitor 4-MP. The negative experience was inferred from a reduction in locomotion and number of falls.

\section{METHOD}

To investigate the effect of previous experience of ingestion of ethanol on the choice of the site of egg laying, flies of the Moulis strain homozygous for the fast allele of the Adh gene (high level of ADH activity) were employed. These flies metabolize alcohol rapidly. The preference for ethanol was examined in flies treated previously with ethanol, 4-MP, or a combination of the two substances. We checked to ensure that avoidance of ethanol in the flies treated with both ethanol and inhibitor was due to the influence on ethanol metabolism and not due to a simple association of ethanol with a repellent substance. Therefore, in a control experiment, we examined whether 4-MP was by itself repellent, neutral, or attractive to the flies. In a further experiment, we looked for signs of behavioral disturbances after ingestion of ethanol, inhibitor, or a combination of the two.

The flies were housed and the experiments were conducted under controlled environmental conditions (temperature $25^{\circ} \mathrm{C}$, humidity $85 \% \pm 5 \%, 12: 12$-h light:dark cycle with 100-lux artificial light). The experimental flies were reared in a regular cornmeal medium without ethanol. This food contained $100 \mathrm{~g}$ corn flour, $20 \mathrm{~g}$ agar, $128 \mathrm{~g}$ sucrose, and $18 \mathrm{~g}$ dried dead yeast in 1 liter of water. To avoid competition between larvae, 25 pairs of progenitors ( $7 \pm 1$ days old) were kept for $24 \mathrm{~h}$ in each bottle containing $100 \mathrm{ml}$ of medium. The absence of ethanol in the medium during the rearing period was checked by gas chromatography (El Amrani, 1991). Under these conditions, the larvae had no early experience of ethanol.

\section{Effect of Experience on Egg-Laying Preference \\ for Ethanol and 4-MP}

At emergence the males and females were separated and placed individually in vials containing regular cornmeal medium without ethanol or inhibitor up to $4 \pm 1$ days of age (age of maximum daily laying; Boulétreau, 1978; David, 1963). After complete starvation (except for water) for $18 \mathrm{~h}$, females were individually kept in the presence of a single male to stimulate egg laying (Boulétreau, 1978; Boulétreau-Merle, 1975; David, 1961, 1963; Hoffmann \& Harshman, 1985) for $24 \mathrm{~h}$ in a vial containing $10 \mathrm{~g}$ of nutriment medium of known composition. Four groups of couples were allocated to the different media for the study of ethanol preference: (1) a cornmeal medium without ethanol or inhibitor (MO), identical to the rearing medium, (2) a cornmeal medium supplemented with $3 \%(\mathrm{w} / \mathrm{v})$ ethanol (MA), (3) a cornmeal medium supplemented with $0.3 \%$ (w/v) 4-MP (MI), and (4) a cornmeal medium containing both ethanol and 4-MP at the above concentrations (MAI).

For the control experiment (behavior of flies toward inhibitor), the flies were placed in contact with MO or MI media.

During the preparation, ethanol and 4-MP were added to regular medium with vigorous stirring, after the medium had been cooled to $50^{\circ} \mathrm{C}$. The concentrations of ethanol and inhibitor were selected on the basis of the results of preliminary experiments. A concentration of $3 \%$ ethanol was found to be favorable to survival of larvae and nymphs and adults, although 0.3\% 4-MP in combination with $3 \%$ ethanol has been shown to reduce the life span of the imagos (El Ghadraoui et al., 1995). In addition, this concentration of inhibitor reduced the activity of $\mathrm{ADH}$ in the same experimental conditions (Cadieu et al., 1999). The concentration of ethanol in the media was also checked at regular intervals (El Amrani, 1991).

After contact for $24 \mathrm{~h}$ with the various media, the females had a choice between the MO and MA media for egg-laying site in the main experiment, and in the control experiment (designed to determine 
the attractant or repellent action of 4-MP) between the MO and the MI media. Each couple was placed in an experimental Plexiglas chamber ( $12 \mathrm{~cm}$ long, $9 \mathrm{~cm}$ wide, and $5 \mathrm{~cm}$ high) for testing the preference of each female. In this chamber, two circular (3-cm diameter) laying dishes were placed in a trough in a sliding plate, enabling daily change of the laying dishes. The flies had access to dishes of food containing MO, MA, or MI depending on the experiment. The choice of egg-laying site was tested over 3 successive days, and the laying dishes were changed and positions reversed every $24 \mathrm{~h}$ to control for a position effect. To check for bias, the flies were also exposed to the same food in the two dishes containing medium without ethanol or inhibitor. There was no significant difference between chance levels of selection of the locus of the two dishes $(50 / 50$; $\chi^{2}=1.38, p>.05$ ). After removal of each couple, the chamber was carefully cleaned with a detergent (Mucapur) to remove all odors.

The experiments were conducted on 180 females with 30 flies per group (4 experimental groups and 2 control groups). Two variables were examined: (1) the total number of eggs laid on the two media, to evaluate the effect of prior exposure to various media on reproductive function, and (2) the percentage of eggs laid on each medium giving an estimate of preference. The number of eggs laid by the females on the two media were counted every day.

For the main experiment (choice between an ethanol-supplemented medium and an alcohol-free medium), we defined for each female a percentage $\left(\mathrm{P}_{\mathrm{A}}\right)$ expressing the preference for ethanol:

$\mathrm{P}_{\mathrm{A}}=\mathrm{N}_{\mathrm{MA}} /\left(\mathrm{N}_{\mathrm{MO}}+\mathrm{N}_{\mathrm{MA}}\right) \times 100$, where

$\mathrm{N}_{\mathrm{MA}}=$ number of eggs laid on ethanol supplemented medium(MA)

$\mathrm{N}_{\text {MO }}=$ number of eggs laid on alcohol-free medium (MO)

We also defined a percentage $P_{I}$ expressing the preference for the inhibitor in the control experiment (choice between $\mathrm{MO}$ and $\mathrm{MI}$ )

Analyses of variance (ANOVAs) were then used for the statistical comparisons. Previous exposures to ethanol and to inhibitor and days (as a repeated measure) were considered in the main experiment and the analysis was followed by post hoc comparisons (Fischer's least significant difference test; Lison, 1968). Previous exposure to inhibitor and days was subjected to analysis in the control experiment.

If ingestion of ethanol was beneficial, the number of laid eggs would be expected to be greater in the MA group than in the MO group. If ingestion of both alcohol and inhibitor reduced reproductive function from inhibition of metabolism, there should be a negative interaction between the effect of ethanol and that of the 4-MP. If the use of ethanol in the fast flies plays a role in preference, the $\mathrm{P}_{\mathrm{A}}$ in the MA group would be expected to be greater than in the MO group. Similarly, if ingestion of both ethanol and 4-MP reduced the preference for ethanol, there should be a negative interaction between the effect of ethanol and that of the inhibitor. The level of significance was $p<.05$.

\section{General Activity of Females:}

\section{Observation of Behavioral Disturbances}

We checked in these experiments whether ethanol and/or 4-MP led to any behavioral disturbances. The locomotor activity and the number of falls were recorded. The female flies for these experiments were housed and reared under identical conditions to those described above. After fasting and being kept for $24 \mathrm{~h}$ in contact with one of the four test diets (MO, MA, MI, or MAI), the flies were introduced individually into an observation chamber $(3 \mathrm{~cm}$ in diameter and $3 \mathrm{~cm}$ high). The floor of the chamber was divided into four sectors around the periphery and a central zone (the central zone was one quarter of the area of the peripheral zone). The walls were also divided into four vertical sectors. The observation chamber was covered with a transparent top. The flies were observed for a period of $10 \mathrm{~min}$ after they had been acclimated for $3 \mathrm{~min}$ to the apparatus. The number of floor and wall sectors crossed was recorded together with the number of falls during movements.
The flies in each of the four groups of 20 flies were observed individually. After removal of each animal, the transparent top was changed and the chamber was cleaned thoroughly with water containing a detergent (Mucapur) and then rinsed with distilled water.

The data were subjected to a two-way ANOVA.

If alcohol combined with inhibitor induced sickness due to metabolic disturbances, there should be a reduction in locomotor activity across the floor and up the walls together with an increase in number of falls, indicating loss of balance. The level of significance was $p<.05$.

\section{RESULTS}

\section{Egg-Laying Behavior Toward Ethanol}

The total number of eggs laid was subjected to a square root conversion (for homogeneity of variances). The ANOVA failed to indicate any significant variations according to the day of the test [repeated measure, $F(2,232)$ $=0.57, \mathrm{n} . \mathrm{s}$. $]$, nor any overall influence of exposure to a medium containing ethanol $[F(1,116)=0.9$, n.s. $]$ when flies had a choice for egg laying between the MO and MA media (Figure 1a). On the other hand, we noted a significant effect of inhibitor $[F(1,116)=45.98, p<.0001]$. The ethanol $\times$ inhibitor interaction $[F(1,116)=13.97, p<$ $.0003]$ revealed a negative influence effect of the combined absorption of these two substances on the number of eggs laid, while the ingestion of ethanol alone favored egg laying (post hoc comparisons using Fischer's least significant difference test: MO vs. MA, $p<.05$ ) and that ingestion of the inhibitor alone had no effect (MO vs. MI, $p>.05$ ).

The percentage $\mathrm{P}_{\mathrm{A}}$ (total number of eggs laid on ethanol/ total number of laid eggs), which assesses the preference for ethanol (Figure 2a) was subjected to an angular transformation arc sine $\sqrt{\mathrm{P} / 100}$ (because the values are proportions). The ANOVA did not evidence any change with days in the choice of egg-laying site $[F(2,232)=1.49$, n.s.], nor any overall influence of ethanol ingestion $[F(1,116)$ $=0.1$, n.s.]. However, absorption of inhibitor affected the preference for ethanol $[F(1,116)=69.02, p<.0001]$. The significant ethanol $\times$ inhibitor interaction $[F(1,116)=$ 49.73, $p<.0001]$ demonstrated a reduction in egg laying on the ethanol-supplemented medium after ingestion of ethanol and inhibitor. However, post hoc comparison (Fischer's least significant difference test) revealed that exposure to ethanol (MA) increased the proportion of eggs laid on this alcohol (comparison MO vs. MA, $p<$ .05 ), while the absorption of inhibitor alone had no effect (MO vs. MI, $p>.05$ ). In the MO and MI groups, the flies were indifferent to ethanol, since the theoretical value arc sine $\sqrt{0.5}$ (chance level: 0.78 radian) was within the confidence interval of the converted value of $\mathrm{P}_{\mathrm{A}}(0.75-0.83$ radian for MO, and 0.71-0.79 radian for MI). Ingestion of ethanol in the MA group induced a preference for this alcohol (0.85-0.95 radian). Treatment with both alcohol and 4-MP (MAI) led to an avoidance (0.58-0.66 radian).

\section{Egg-Laying Behavior Toward the Inhibitor}

The ANOVA (after square root conversion of the values) failed to evidence any significant influence of pre- 
(a)

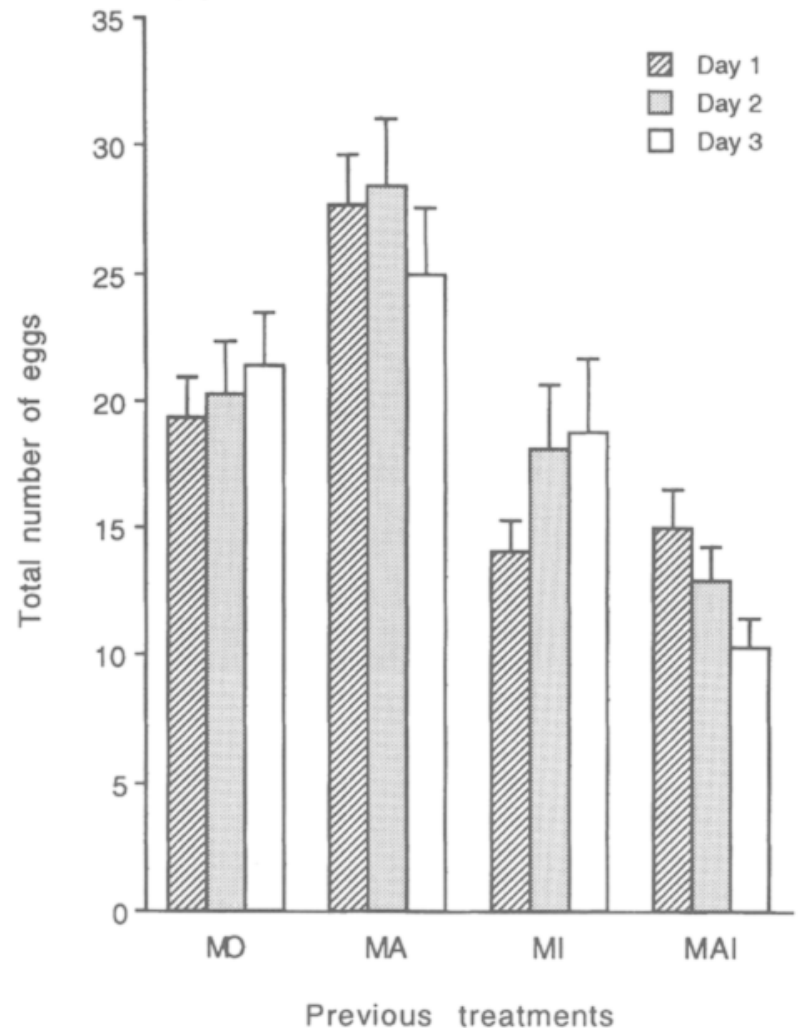

(b)

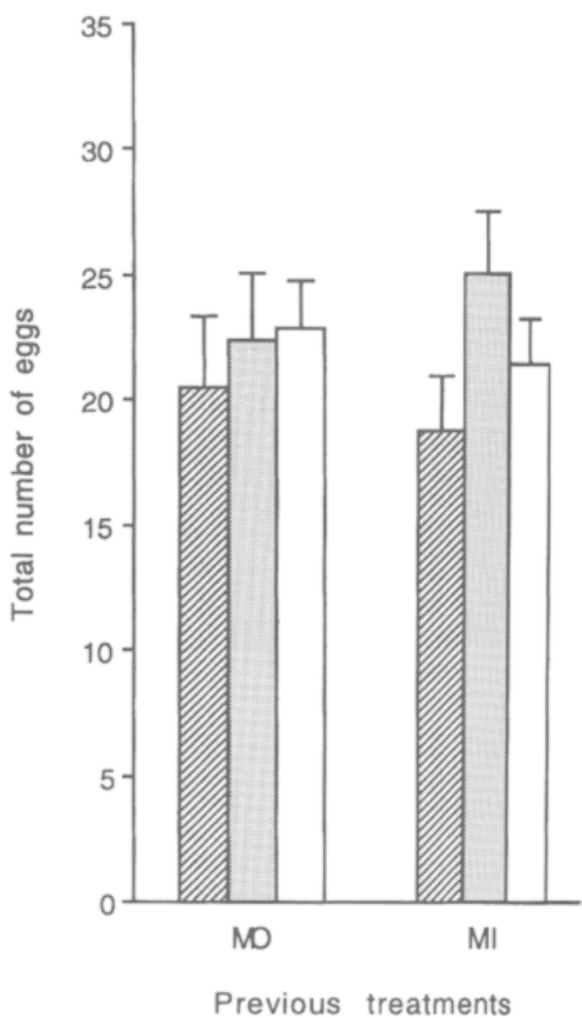

Figure 1. Mean number ( $\pm S E$ ) of eggs laid over 3 successive days in female Drosophila that had the choice between an ethanol-supplemented medium and an alcohol-free medium (a) or between an inhibitor-supplemented medium and a medium without inhibitor (b). The flies were previously kept in contact with an alcohol-free medium (MO), a medium supplemented with $3 \%$ (w/v) ethanol (MA), a medium supplemented with $0.3 \%$ (w/v) 4-methylpyrazole (MI), or a medium containing ethanol and inhibitor (MAI). Analysis after square root conversion indicated an increase in the number of eggs laid after treatment with ethanol (MA) and a reduction after treatment with both ethanol and inhibitor (MAI).

vious exposure to inhibitor on the total number of eggs laid (Figure 1b) when flies had the choice between MO and $\mathrm{MI}[F(1,58)=0.03$, n.s. $]$, nor any change of number of eggs laid with days $[F(2,116)=2.65$, n.s.].

Exposure to the inhibitor increased the percentage (Figure 2b) of eggs laid on 4-MP [ANOVA after angular transformation, $F(1,58)=29.2, p<.0001]$, but the choice was not altered with the days of the test $[F(2,116)=0.34$, n.s.]. The flies without experience of inhibitor $(\mathrm{MO})$ were indifferent to it (theoretical value arc sine $\sqrt{0.5}$ within the confidence interval: $0.76-0.82$ radian). Prior exposure to 4-MP induced a preference for it (confidence limits: $0.86-0.95$ radian).

\section{General Activity of Flies and \\ Behavioral Disturbances}

Analysis of the number of sectors crossed on the floor and on the wall (after square root conversion of the values) of the observation chamber demonstrated a reduction in locomotor activity (Figure 3a) in flies that had absorbed both ethanol and inhibitor. The ANOVA revealed an overall influence of treatment with ethanol $[F(1,76)=$
$4.4, p<.05]$ and with inhibitor $[F(1,76)=9.6, p<.05]$, as well as an interaction between the effects of ethanol and inhibitor $[F(1,76)=14.28, p<.05]$. However, post hoc comparisons failed to show any influence of the ingestion of ethanol alone (MO vs. MA, n.s.) or of inhibitor alone (MO vs. MI, n.s.).

Loss of balance as indicated by falls was observed in the flies exposed to both ethanol and inhibitor (Figure 3b). An ANOVA (after square root transformation) showed an overall influence of exposure to ethanol $[F(1,76)=$ $11.98, p<.05]$ and to inhibitor $[F(1,76)=16.94, p<.05]$. However, the ingestion of these substances taken separately had no effect (MO vs. MA, n.s., and MO vs. MI, n.s.). The increased loss of balance was observed after exposure to a combination of the two substances (significant ethanol $\times$ inhibitor interaction $[F(1,76)=7.88, p<.05]$.

\section{DISCUSSION}

The results showed that experience of ethanol had an influence on reproductive function and on the preference of Drosophila melanogaster for laying eggs on an ethanol 
(a)

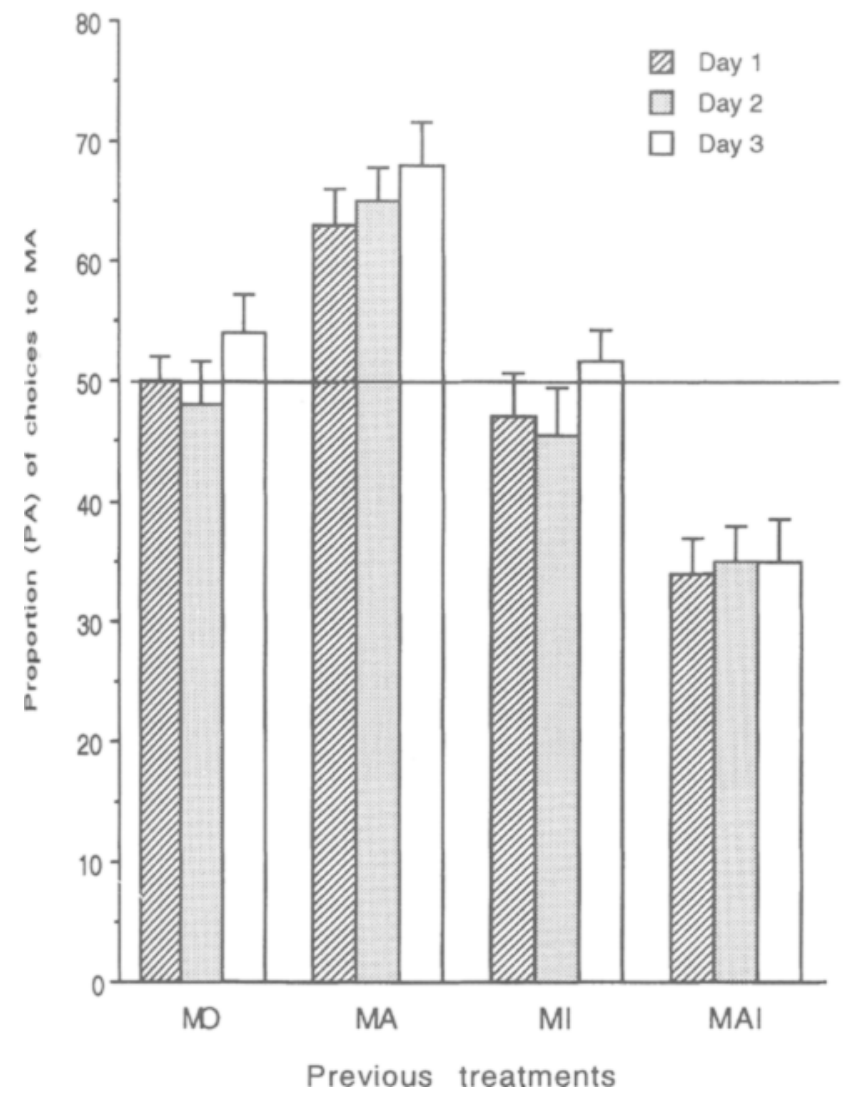

(b)

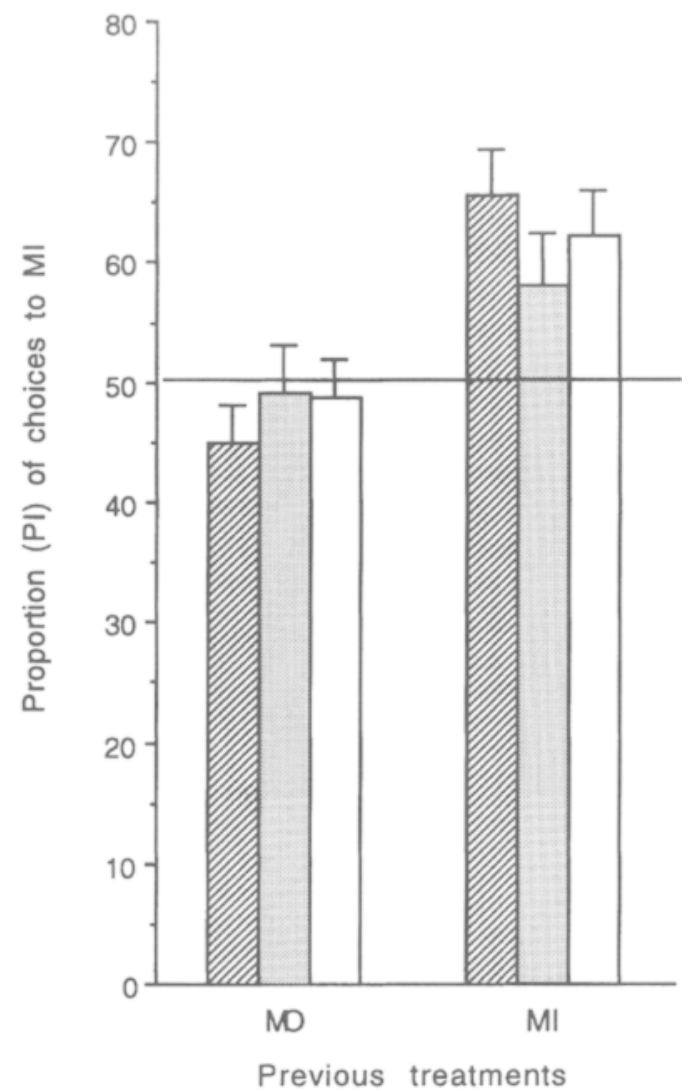

Figure 2. Mean percentage $( \pm S E)$ of eggs laid over 3 successive days on an ethanol-supplemented medium $\left(P_{A}\right)$ or an inhibitorsupplemented medium $\left(P_{I}\right)$ in female Drosophila that had the choice between an ethanol-supplemented medium and an alcohol-free medium (a) or between an inhibitor-supplemented medium and a medium without inhibitor (b). The horizontal line indicates indifference to ethanol or to inhibitor. The flies were previously kept in contact with an alcohol-free medium (MO), a medium supplemented with $3 \%$ (w/v) ethanol (MA), a medium supplemented with $0.3 \%$ (w/v) 4-methylpyrazole (MI), or a medium containing ethanol and inhibitor (MAI). Analysis after angular conversion indicated the appearance of a preference for ethanol after treatment with it (MA) and of an aversion after treatment with both ethanol and inhibitor (MAI).

substrate. The flies with the fast form of the Adh gene were indifferent to low doses of ethanol for egg-laying site in the absence of previous experience of this substance, in spite of their high-level activity of the ADH enzyme, which degrades ethanol.

Prior contact with ethanol led to an increase in number of eggs laid, and so low doses of this alcohol acted as a food (Cadieu et al., 1997; David et al., 1976; DeltombeLietaert et al., 1979; El Ghadraoui et al., 1995; Heinstra, 1993). Its ingestion appeared to enhance the motivation to lay eggs. Our observations also suggested a preference for alcohol that was related to its ingestion on the egglaying medium. This alteration of preferences stemmed from learning. The putative learning process involved in this case could not be attributed to simple habituation to ethanol (as observed by Jaenike, 1982, with high doses), because we observed a preference for this substance, whereas habituation would tend to reduce the response to the stimulus (Davis, 1996; Papaj \& Prokopy, 1989). The choice of an alcohol-containing medium as a site for egg laying was not accompanied by an induction of ADH. It has been shown that the high activity of this enzyme in adult fast Drosophila without prior exposure to ethanol is not increased after ingestion of this alcohol (Barbancho, 1992; Cadieu et al., 1999).

However, there is probably a link between the benefit obtained by absorption of ethanol and the acquired preference for it. This was borne out by the effect observed of ingestion of ethanol and 4-MP, an inhibitor of ADH. With doses of 4-MP that inhibit ADH (Cadieu et al., 1999), the flies had a general reduction in reproductive function accompanied by an acquired avoidance of ethanolcontaining media for egg laying. These results did not stem from an action of inhibitor alone, which did not reduce the number of eggs laid nor modify the preference for ethanol over the test period (Figure 2). We concluded 


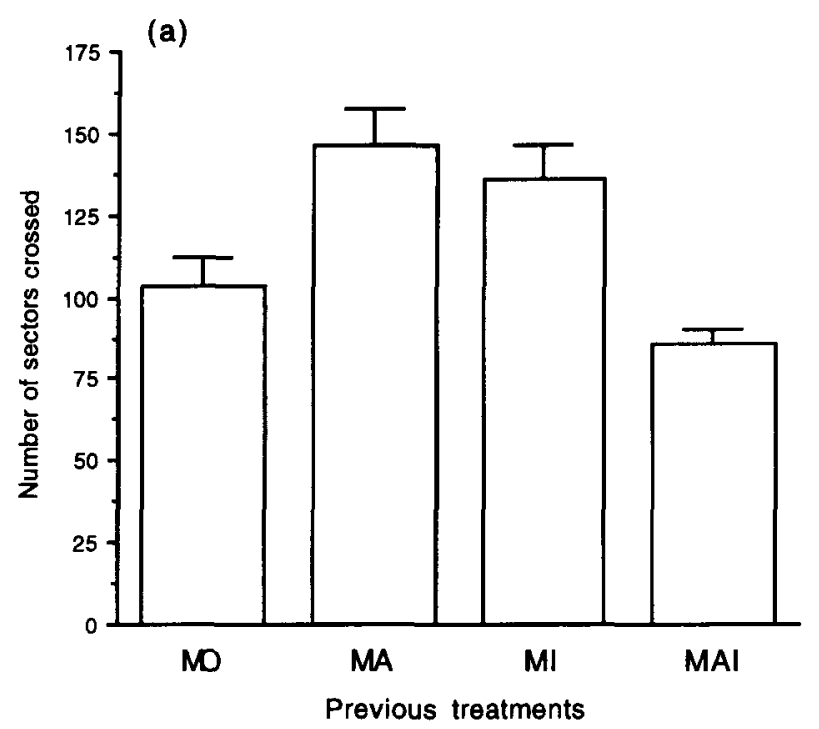

(b)

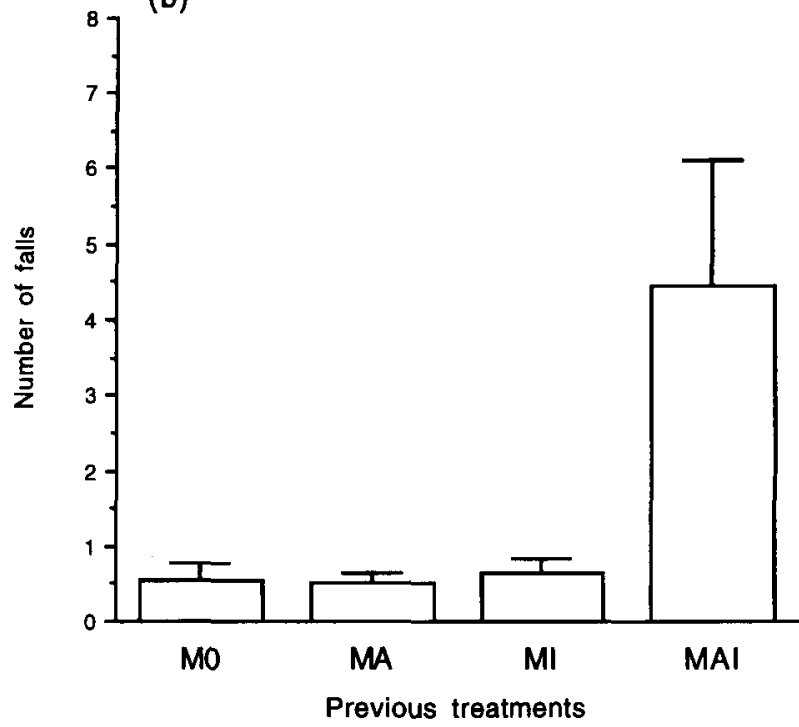

Figure 3. Locomotor activity and number of falls recorded for a period of $10 \mathrm{~min}$ in female Drosophila previously kept in contact with an alcohol-free medium (MO), a medium supplemented with $3 \%$ (w/v) ethanol (MA), a medium supplemented with $0.3 \%$ (w/v) 4-methylpyrazole (MI), or a medium containing ethanol and inhibitor (MAI). (a) mean number $( \pm S E)$ of sectors crossed on the floor and the wall of the observation chamber. (b) mean number $( \pm S E)$ of falls. Analysis after square root conversion indicated a reduction of locomotor activity and an increase in falls after prior treatment with both ethanol and inhibitor.

that the avoidance of ethanol throughout the test by the flies treated with ethanol and 4-MP was due to learning followed by memory.

We noticed also that 4-MP itself had a slight attractant (Figure 2b) action, and so the avoidance for ethanol could not have been due to association with a repellent substance. The modification in preference was thus assumed to be linked to the disturbance of ethanol metabolism via the
ADH pathway, such as an accumulation of ethanol in tissues. The metabolic disturbance was reflected by signs of sickness analogous to those observed in caterpillars after ingestion of toxic food (Dethier, 1980). Most of the flies treated with ethanol and 4-MP exhibited a reduction in locomotor activity and loss of balance. Under these conditions, the ingestion of ethanol before the tests appeared to be followed by a punishment, which led to avoidance of ethanol-containing media during the test. These observations are consistent with those in insects that ingestion of harmful food leads to aversion for them (Cadieu et al., 1999; Dethier, 1980; Papaj \& Prokopy, 1989). True conditioned aversion has also been demonstrated in insects (Bernays \& Lee, 1988).

The fly adjusts its response after first contact with ethanol as a function of its level of ADH activity, which governs its ability to use this substance as food. Modification of preference for egg-laying site is thus a result of learning, which can be considered to be an adaptive change due to experience (Thorpe, 1963) since the eggs are laid on a medium that leads to the highest fecundity. Furthermore, the food preference of fast Drosophila for ethanol is enhanced by prior ingestion of this substance, whereas absorption of ethanol associated with an inhibitor leads to avoidance of ethanol as food (Cadieu et al., 1999). In this respect, it has been shown that flies are longer lived when reared on a medium containing $3 \%(\mathrm{w} / \mathrm{v})$ ethanol than controls reared on the same medium devoid of ethanol. Our group has also shown that rearing on a medium containing both ethanol and 4-MP shortened the life span (El Ghadraoui et al., 1995). In sum, experienced adult Drosophila choose via a process of learning to feed and to lay eggs on media that give rise to the greatest fecundity and life span, which are the major components of fitness (Dobzhansky, 1956).

Although the role of learning in choice of food and egg-laying site has been demonstrated in a large number of insects, its adaptive value is difficult to establish (Dukas, 1999; Papaj \& Prokopy, 1989). The role of ADH in the adaptation of Drosophila to the presence of ethanol and the environment is the subject of debate (Briscoe, Robertson, \& Malpica, 1975; McKenzie, McKehnie, \& Batterham, 1994; McKenzie \& Parsons, 1974; Oakeshott et al., 1982). It would seem, however, that this adaptation can be comprehended only by considering the role of behavior, and of learning, in particular, which enables larvae and adult flies to settle in microhabitats of differing levels of alcohol content according to relative fitness (Powell \& Taylor, 1979).

Barbancho (1992) has argued that larvae Drosophila adapt to the alcohol content of the medium by altering their enzymatic activity, while adults tend to move toward substrates that are compatible with their tolerance to ethanol. We have shown that adaptation to the presence of ethanol results in learning in the adults with respect to both choice of food source (Cadieu et al., 1999) and egglaying site. In a previous study, we showed that larvae also selected alcohol-containing media as a function of their 
genetic ability to degrade ethanol. Rearing on the selected medium favored larvo-nymphal survival and produced heavier imagos (Cadieu et al., 1997). The Drosophila thus retained the benefit of their choice as larvae.

It is known that an increase in weight is accompanied by a selective advantage of adults, as reflected by the increase in number of eggs laid (Tantawy \& Vetukliv, 1960) and the greater success in sexual competition by the males (Partridge, Ewing, and Chandler, 1987). Dukas (1998), employing the methodology of Tully, Cambiazo, and Kruse (1994), has shown that choice behavior by Drosophila larva of adaptive significance stems from associative learning. However, Papaj and Prokopy (1989) have pointed out that acceptance of a food source under natural conditions will involve a variety of learning processes. Drosophila thus appear to adapt to changing environmental conditions by learning throughout life as a function of their individual capacities. Study of learning in Drosophila melanogaster thus opens interesting perspectives because it has adaptive value and is thus involved in natural selection. Furthermore, in this species, the genetic architecture is largely known and genes involved in learning process have been identified.

\section{REFERENCES}

BARBANCHO, M. (1992). Effect of dietary acetaldehyde 2 propanol and acetone on the variation of several enzyme activities involved in alcohol metabolism of Drosophila melanogaster adults. Insect Biochemistry \& Molecular Biology, 22, 269-276.

BERNAYS, E. A., \& LEE, J. C. (1988). Food aversion learning in the polyphagous grasshopper Schistocerca americana. Physiological Entomology, 13, 131-137.

Blaney, N. M., \& Simmonds, M. S. J. (1985). Food selection by locusts: The role of learning in rejection behaviour. Entomologia experimentalis et applicata, 39, 273-278.

BoulÉTreAU, J. (1978). Ovarian activity and reproductive potential in a natural population of Drosophila melanogaster. OEcologia, 35, 319-342.

BoulÉtreau-MerLe, J. (1975). Influence de l'accouplement sur la physiologie des femelles de Drosophila melanogaster (Meig): Fonctions modifiées, nature des stimulations reçues et relations physiologiques mises en jeu. Unpublished doctoral dissertation, University of Lyon.

Briscoe, D. A., Robertson, A., \& Malpica, M. J. (1975). Dominance at $\mathrm{ADH}$ locus in response of adult Drosophila melanogaster to environmental alcohol. Nature, 255, 148-149.

Cadieu, N., Cadieu J.-C., El Ghadraoui, L., Grimal, A., \& LamBoEUF, Y. (1999). Conditioning to ethanol in the fruit fly-A study using an inhibitor of ADH. Journal of Insect Physiology, 28, 579-586.

Cadieu, N., El Amrani, A., \& Cadieu, J.-C. (1997). Conséquences des préférences larvaires pour l'éthanol sur les stades préimaginaux et adultes de Drosophila melanogaster. Annales des Sciences naturelles, 18, $135-149$.

Cavener, D. (1979). Preference for ethanol in Drosophila melanogaster associated with the alcohol dehydrogenase polymorphism. Behavior Genetics, 9, 359-366.

DAvid, J. (1961). Étude quantitative du fonctionnement ovarien chez Drosophila melanogaster Meig. Bulletin Biologique de la France et de la Belgique, 3, 522-535.

DAviD, J. (1963). Influence de la fécondation de la femelle sur le nombre et la taille des oeufs pondus: Étude chez Drosophila melanogaster Meig. Journal of Insect Physiology, 9, 13-24.

DAvID, J. (1977). Significance of enzymatic polymorphism alcohol dehydrogenase (EC-1.1.1.1) in Drosophila melanogaster. L'Année biologique, 16, 451-472.
David, J. (1978). Du génotype au phénotype. La Recherche, 9, 482-483. DAVID, J., \& BocqueT, C. (1975). Similarities and differences in latitudinal adaptation of two Drosophila sibling species. Nature, 257, 588-590.

David, J., Bocquet, C., Arens, M. F., \& Foulllet, P. (1976). Biological role of alcohol dehydrogenase in the tolerance of Drosophila melanogaster to aliphatic alcohols: Utilization of an ADH-null mutant. Biochemical Genetics, 14, 989-997.

Davis, R. L. (1996). Physiology and biochemistry of Drosophila learning mutants. American Physiological Society, 76, 299-317.

Delmas, C. (1981). Nouveaux inhibiteurs de l'ADH: Effets biochimiques et physiopathologiques lors de divers traitements éthanoliques chez le rat. Unpublished doctoral dissertation, Université Paul Sabatier.

Deltombe-Lietaert, M. C., Delcour, J., Lenelle-Monfort, N., \& ELENS, A. (1979). Ethanol metabolism in Drosophila melanogaster. Experientia, 35, 579-581.

DETHIER, V. G. (1980). Food aversion learning in two polyphagous caterpillars Diacrisia virginica and Estigmene congrua. Physiological Entomology, 5, 321-325.

DoBZHANSKY, T. H. (1956). What is an adaptative trait? American Naturalist, 90, 337-347.

DUDAI, Y. (1977). Properties of learning and memory in Drosophila melanogaster. Journal of Comparative Physiology, 114, 69-89.

DuKAS, R. (1998). Ecological relevance of associative learning in fruit fly larvae. Behavioral Ecology \& Sociobiology, 19, 195-200.

DuKas, R. (1999). Cost of memory: Ideas and predictions. Journal of Theoretical Biology, 197, 41-50.

Dura, J. M., Prat, T., \& Tully, T. (1993). Identification of Linotte, a new gene affecting learning and memory in Drosophila melanogaster. Journal of Neurogenetics, 9, 1-14.

El Amrani, A. (1991). Étude expérimentale des facteurs génétiques et épigénétiques de la variabilité inter-individuelle du comportement du choix de l'habitat chez Drosophila melanogaster. Unpublished doctoral dissertation, University of Paul Sabatier.

El Amrani, A., CAdieu, N., \& Cadieu, J.-C. (1991). Influence d'un conditionnement pré-imaginal par l'alcool sur le choix du substrat alimentaire et du site de ponte par les adultes de Drosophila melanogaster. Behavioural Processes, 24, 153-165.

El Ghadraoui, L., Cadieu, N., \& Cadieu, J.-C. (1995). Inhibition de l'ADH et longévité en milieu alcoolisé de mouches fast et slow: Étude chez Drosophila melanogaster. Annales des Sciences Naturelles, Zoologie, 16, 113-119.

FuYAMA, Y. (1976). Behavior genetics of olfactory responses in Drosophila. I: Olfactory responses and strain differences in Drosophila melanogaster. Behavior Genetics, 6, 407-419.

Heinstra, P. W. H. (1993). Evolutionary genetics of the Drosophila alcohol dehydrogenase gene-enzyme system. Genetica, 92, 1-22.

Heinstra, P. W. H., \& GeER, B. W. (1991). Metabolic control analysis and enzyme variation nutritional manipulation of the flux from ethanol to lipids in Drosophila. Molecular Biology \& Evolution, 8, 703-708.

Heinstra, P. W. H., Geer, B. W., Seykens, D., \& Langevin, M. (1989). The metabolism of ethanol-derived acetaldehyde by alcohol dehydrogenase (EC 1.1.1.1) and aldehyde dehydrogenase (EC 1.2.1.3) in Drosophila melanogaster larvae. Biochemical Journal, 259, 791-797.

Hennemann, M. L., Papaj, D. R., Figueredo, A. J., \& Vet, L. E. M. (1995). Egg-laying experience and acceptance of parasitized host by the parasitoid, Leptopilina heterotoma (Hymenoptera: Eucoilidae). Journal of Insect Behavior, 8, 331-342.

Hoffmann, A. A. (1985). Effects of experience on oviposition and attraction in Drosophila: Comparing apples and oranges. American Naturalist, 126, 41-51.

HoffMANN, A. A. (1988). Early adult experience in Drosophila melanogaster. Journal of Insect Physiology, 34, 197-204.

Hoffmann, A. A., \& Cohan, F. M. (1987). Olfactory response of Drosophila melanogaster selected for knockdown resistance to ethanol. Behavior Genetics, 17, 307-310.

Hoffmann, A. A., \& Harshman, L. G. (1985). Male effects on fecundity in Drosophila melanogaster. Evolution, 39, 638-644.

Hougouto, N., Lietaert, M. C., Libion-Mannaert, M., FeytMANS, E., \& ELENS, A. (1982). Oviposition site preference and alcohol dehydrogenase activity in Drosophila melanogaster. Genetica, 58, 121-128. 
IIZUKA, T., \& TAKASU, K. (1998). Olfactory associative learning of the pupal parasitoid Pimpla luctuosa Smith (Hymenoptera: Ichneumonidae). Journal of Insect Behavior, 11, 743-760.

JAENIKE, J. (1982). Environmental modification of oviposition behavior in Drosophila. American Naturalist, 119, 784-802.

KAISER, L., \& DE JONG, R. (1995). Induction of odor preference in a specialist insect parasitoid. Animal Learning \& Behavior, 23, 17-21.

Lewis, W. J., \& TumLinson, J. H. (1988). Host detection by chemically mediated associative learning in a parasitic wasp. Nature, 331, 257-259.

Lison, L. (1968). Statistiques appliquées à la biologie expérimentale. Paris: Gauthier-Villars.

Maynard-Smith, J. (1966). Sympatrie speciation. American Naturalist, 100, 637-650.

McKenzie, J. A., McKehnie, S. W., \& Batterham, P. (1994). Perturbation of gene frequencies in a natural population of Drosophila melanogaster: Evidence for selection at the ADH locus. Genetica, 92, 187-196.

McKenzie, J. A., \& Parsons, P. A. (1972). Alcohol tolerance: An ecological parameter in the relative success of Drosophila melanogaster and Drosophila simulans. OEcologia, 10, 373-388.

McKenzie, J. A., \& Parsons, P. A. (1974). Microdifferentiation in a natural population of Drosophila melanogaster to alcohol in the environment. Genetics, 77, 385-394.

MÉDIONI, J., CADIEU, N., \& VAYsSE, G. (1978). Sélection divergente pour la rapidité d'acquisition d'un conditionnement chez la drosophile (Drosophila melanogaster). Comptes rendus de la Société de Biologie, 172, 961-967.

OAKEShott, J. G., Gibson, J. B., Anderson, P. R., KnibB, W. R., ANDERSON, D. G., \& ChAmbers, G. K. (1982). Alcohol dehydrogenase and glycerol-3-phosphate dehydrogenase clines in Drosophila melanogaster on different continents. Evolution, 36, 86-96.

PAPAJ, D. R. (1986). Conditioning of leaf-shape discrimination by chemical cues in the butterfly, Battus philenor. Animal Behaviour, 34, 1281-1288.

PAPAJ, D. R., \& Prokopy, R. J. (1989). Ecological and evolutionary aspects of learning in phytophagous insects. Annual Review of Entomology, 34, 315-350.

Partridge, L., Ewing, A., \& Chandler, A. (1987). Male size and mat- ing success in Drosophila melanogaster: The roles of male and female behaviour. Animal Behaviour, 35, 555-562.

POWELL, J. R., \& TAYLOR, C. E. (1979). Genetic variation in ecologically diverse environments. American Scientist, 5, 590-596.

Prokopy, R. J., Averill, A. L., Cooley, S. S., \& Roitberg, C. A. (1982). Associative learning in egg-laying site selection by apple maggot flies. Science, 218, 76-77.

TANTAWY, A. O., \& VetUKLIV, M. O. (1960). Effects of size on fecundity, longevity and viability in populations of Drosophila pseudoobscura. American Naturalist, 94, 395-403.

THORPE, W. H. (1963). Learning and instinct in animals. London: Methuen.

Traynier, R. M. M. (1984). Associative learning in the ovipositional behavior of the cabbage butterfly Pieris rapae. Physiological Entomology, 9, $465-472$

Tully, T. (1996). Discovery of genes involved with learning and memory: An experimental synthesis of Hirschian and Benzerian perspectives. Proceedings of the National Academy of Sciences, 93, 1346013467.

Tully, T., Cambiazo, V., \& Kruse, L. (1994). Memory through metamorphosis in normal and mutant Drosophila. Journal of Neurosciences, 14, 68-74.

TulLy, T., \& QuinN, W. G. (1985). Classical conditioning and retention in normal and mutant Drosophila melanogaster. Journal of Comparative Physiology, 157, 263-277.

VAN DELDEN, W., \& KAMPING, A. (1990). Genetic variation for oviposition behavior in Drosophila melanogaster. II-Oviposition preferences and differential survival. Behavioral Genetics, 20, 661-674.

VET, L. E. M. (1983). Host-habitat location through olfactory cues by Leptopilina clavipes (Hartig) (Hym.: Eucoilidae), a parasitoid of fungivorous Drosophila: The influence of conditioning. Netherlands Journal of Zoology, 33, 225-248.

WARdLe, A. R., \& Borden, J. H. (1985). Age-dependent associative learning by Exeristes roborator (F.) (Hymenoptera: Ichneumonidae). Canadian Entomologist, 117, 605-616.

(Manuscript received May 24, 1999; accepted for publication October 22, 1999.) 